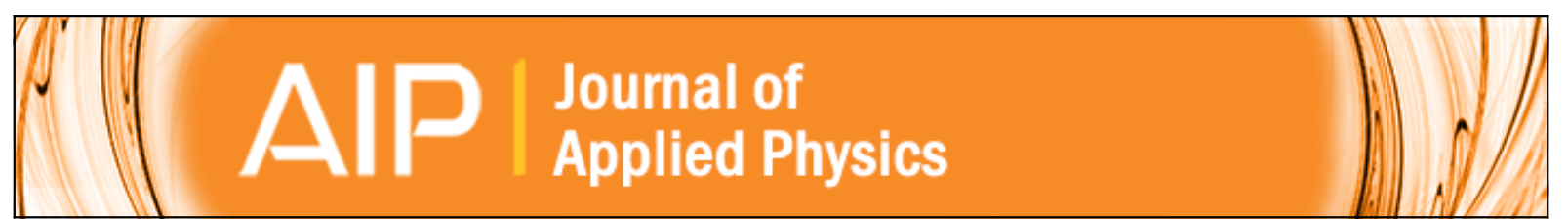

Favorable electronic structure for organic solar cells induced by strong interaction at interface

Shenghao Wang, Takeaki Sakurai, Xia Hao, Wei Fu, Shigeru Masuda, and Katsuhiro Akimoto

Citation: Journal of Applied Physics 114, 183707 (2013); doi: 10.1063/1.4829905

View online: http://dx.doi.org/10.1063/1.4829905

View Table of Contents: http://scitation.aip.org/content/aip/journal/jap/114/18?ver=pdfcov

Published by the AIP Publishing

$\underset{\substack{\text { Publoning } \\ \text { PIP }}}{A}$ Re-register for Table of Content Alerts

Create a profile.

Sign up today! 


\title{
Favorable electronic structure for organic solar cells induced by strong interaction at interface
}

\author{
Shenghao Wang, ${ }^{1, a)}$ Takeaki Sakurai,,${ }^{1,2}$ Xia Hao, ${ }^{1}$ Wei Fu, ${ }^{1}$ Shigeru Masuda, ${ }^{3}$ \\ and Katsuhiro Akimoto ${ }^{1}$ \\ ${ }^{1}$ Institute of Applied Physics, University of Tsukuba, Tsukuba, Ibaraki 305-8573, Japan \\ ${ }^{2}$ PRESTO, Japan Science and Technology Agency (JST), Kawaguchi, Saitama 332-0012, Japan \\ ${ }^{3}$ Department of Basic Science, Graduate School of Arts and Sciences, The University of Tokyo, Komaba, \\ Meguro, Tokyo 153-8902, Japan
}

(Received 9 September 2013; accepted 26 October 2013; published online 12 November 2013)

\begin{abstract}
To clarify the role of buffer layer in organic solar cells (OSCs), the electronic properties of bathocuproine (BCP)/Mg interface were systematically investigated by using ultraviolet photoemissions spectroscopy, high-resolution X-ray photoemission spectroscopy, angle-resolved $\mathrm{X}$-ray photoemission spectroscopy and near-edge X-ray absorption fine structure (NEXAFS) spectroscopy. The results show there are gap states at the interface, which are caused by the interaction between $\mathrm{BCP}$ and $\mathrm{Mg}$. The formation of $\mathrm{Mg}-\mathrm{N}$ bond was found at the interface. The NEXAFS measurements show that BCP molecules for 1-2 monolayers are lying-down on the substrate, whereas orient randomly for thick BCP layer. It was supposed that the gap states and the highly-ordered orientation of thin BCP layer are the reasons for improving the performance of OSC with BCP buffer layer and low work function metal cathode. (C) 2013 AIP Publishing LLC. [http://dx.doi.org/10.1063/1.4829905]
\end{abstract}

\section{INTRODUCTION}

Organic solar cells (OSCs) have great potential in the photovoltaic market due to the advantages of low cost, lightweight, easy fabrication, and flexibility. ${ }^{1,2}$ A buffer layer, such as $\mathrm{LiF}$, bathocuproine $\left(\mathrm{C}_{26} \mathrm{H}_{20} \mathrm{~N}_{2}\right.$, known as $\left.\mathrm{BCP}\right)$, $\mathrm{Alq}_{3}, \mathrm{MoO}_{3}, \mathrm{Cs}_{2} \mathrm{CO}_{3}, \mathrm{ZnO}$, and $\mathrm{TiO}_{2}$, plays an important role in enhancing the performance of OSCs. ${ }^{3-9}$ Among them, the most popular one is $\mathrm{BCP}$, since it can greatly improve the power conversion efficiency $(P C E)$ as compared to others. ${ }^{10,11}$ Fig. 1 shows the chemical structure of BCP molecule. Primarily, BCP was regarded as an exciton-blocking layer. ${ }^{4}$ However, other interpretations were also proposed to explain the role of BCP, such as increasing the built-in field, acting as an optical spacer, reducing nonradiative recombination of excitons at the fullerene $\left(\mathrm{C}_{60}\right) / \mathrm{Al}$ interface, avoiding the formation of aluminum carbide, and so on. ${ }^{12,13}$ Anyway, the working mechanism is still controversial.

Generally, a low work function $(W F)$ metal is desirable to be the cathode in OSCs, because it can increase the electric built-in field, and therefore effectively extract free charges. However, we found the electronic properties of $\mathrm{BCP} /$ metal interface are strongly dependent on the metal's $W F$ by forming different dipole layers. ${ }^{13}$ Moreover, it has been demonstrated that the energy level alignment of $\mathrm{C}_{60} / \mathrm{BCP} / \mathrm{Ag}$ interfaces can be drastically influenced by BCP layer thickness. ${ }^{12}$ This suggests that the increase of electric built-in field is not the only reason for the efficiency enhancement for OSC with BCP and low $W F$ cathode, and other reasons should be considered.

To further improve the PCE of OSCs, it is essential to investigate the electronic properties of $\mathrm{BCP} /$ low $W F$ metal interface, because the interfacial electronic properties of

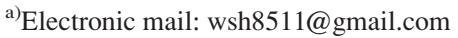

organic electronics may affect the energy level alignment, charge transfer, barrier height, and so on. In this study, $\mathrm{Mg}$ was used to be the metal, which has a low $W F$ of $3.7 \mathrm{eV}$. The electronic properties of $\mathrm{BCP} / \mathrm{Mg}$ interface was systematically studied by ultraviolet photoemissions spectroscopy (UPS), high-resolution X-ray photoemission spectroscopy (HR-XPS), angle-resolved X-ray photoemission spectroscopy (ARPES), and near-edge X-ray absorption fine structure (NEXAFS) spectroscopy. It was found that there are strong interactions between $\mathrm{BCP}$ and $\mathrm{Mg}$, and this interaction can result in a favorable electronic structure for OSCs.

\section{EXPERIMENTS}

In the experiments, ex situ cleaned Si (100) wafers were used as substrates. The Mg layer and organic films were

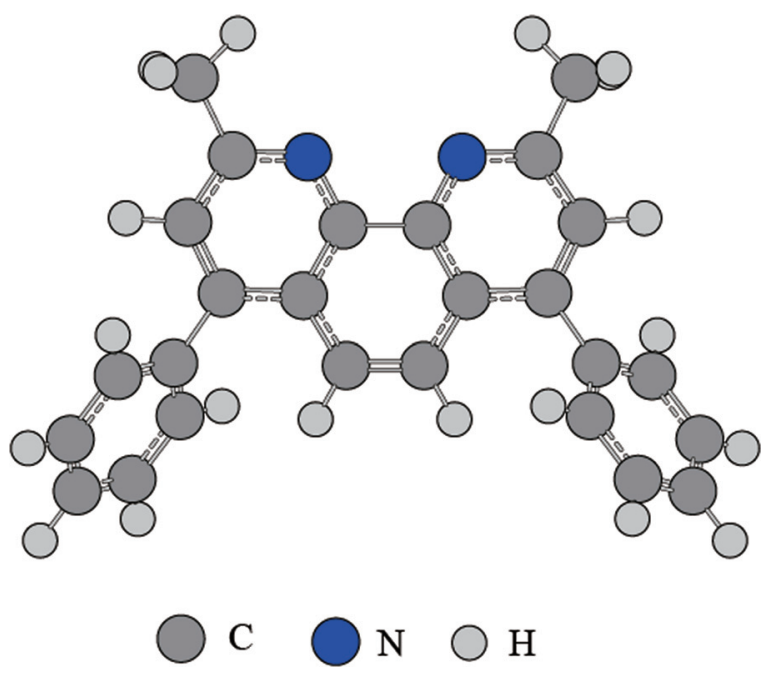

FIG. 1. The chemical structure of BCP molecule. 
deposited by thermal evaporation in ultra-high vacuum chambers $\left(<8.0 \times 10^{-9}\right.$ Torr $)$. The thickness was monitored by a quartz-crystal microbalance. Prior to deposition, BCP (Wako Corp., sublimated grade) and $\mathrm{C}_{60}$ (Sigma-Aldrich, $99.5 \%$ ) were purified three times by vacuum gradient sublimation to avoid any impurities. The deposition chamber is connected to an ultra-high analysis chamber. After each deposition step for a certain layer, the sample was immediately transferred to the analysis chamber to get the spectra without breaking the vacuum.

UPS experiment was performed at Beam-line 3B of KEK-PF in Tsukuba, Japan. The spectra were measured using photon energy of $30 \mathrm{eV}$. The experiments of HR-XPS, ARPES, and NEXAFS were carried out at Beam-line $13 \mathrm{~A}$ of KEK-PF using soft $\mathrm{X}$-ray. The XPS spectra were recorded by a hemispherical electron energy analyzer (Scienta, SES$200)$ in an ultrahigh vacuum chamber $\left(<5.0 \times 10^{-10}\right.$ Torr $)$. Synchrotron radiation entered the chamber at an angle of $65^{\circ}$ with respect to the analyzer lens axis with horizontally linear polarized light. The $\mathrm{C} 1 \mathrm{~s}$ and N1s core level spectra were collected at photon energy of $h v=405$ and $520 \mathrm{eV}$, respectively. To avoid the contamination, the $\mathrm{Si}$ substrate was heated at about $900{ }^{\circ} \mathrm{C}$ before any deposition, and thereafter the cleanliness of substrate was checked by XPS spectra (C1s) till no observation of carbon peak. For the ARPES measurements, the sample surface was oriented normal or $25^{\circ}$ from the analyzer. The angular-dependent NEXAFS spectra were performed by the total electron yield (TEY) method with an MCP detector set at a retarding voltage of $-1800 \mathrm{~V}$. The incidence angle of the light with respect to the sample surface was changed from normal to grazing $\left(25^{\circ}\right.$ off normal). The spectra were normalized to the current from a gold coated mesh positioned in the incident photon beam. To avoid the damage of the organic layer, the measurement point was shifted to a fresh position after each scan.

\section{RESULTS AND DISCUSSION}

\section{A. BCP/Mg interfacial properties studied by UPS}

Fig. 2 shows UPS spectra of BCP layers with incremental thicknesses on $\mathrm{Mg} / \mathrm{Si}$ substrate at the low binding energy $(B E)$ part near the Fermi level $\left(E_{F}\right)$. The abscissa is the $B E$ relatively to the $E_{F}$ of a clean $\mathrm{Mg}$ sample. After depositing $\mathrm{BCP}$, small peaks located at about 0.5 and $2.5 \mathrm{eV}$, which are labeled with $H_{1}$ and $H_{2}$, can be clearly observed. With increasing BCP layer thickness from 0.2 to $1.6 \mathrm{~nm}$, the intensity of peak $H_{1}$ decreased, whereas the intensity of peak $H_{2}$ increased. Above $1.6 \mathrm{~nm}$, the peak $H_{1}$ and peak $H_{2}$ disappeared and the spectra were dominated by bulk BCP features. These two peaks represent the presence of new states, namely, gap states at the interface, because they can only be discerned for thin BCP layer, rather than thick layer. Theoretical calculation also shows two gap states exist when $\mathrm{BCP}$ molecule is absorbed on $\mathrm{Mg}$, being consistent with the experimental UPS results. ${ }^{15}$

On the other hand, the peak $H_{1}$ shows no shift, whereas the peak $\mathrm{H}_{2}$ shifted to lower $B E$. The different position and evolution of these peaks indicate that the origins of gap states are different. It seems as if the peak $H_{1}$ is the

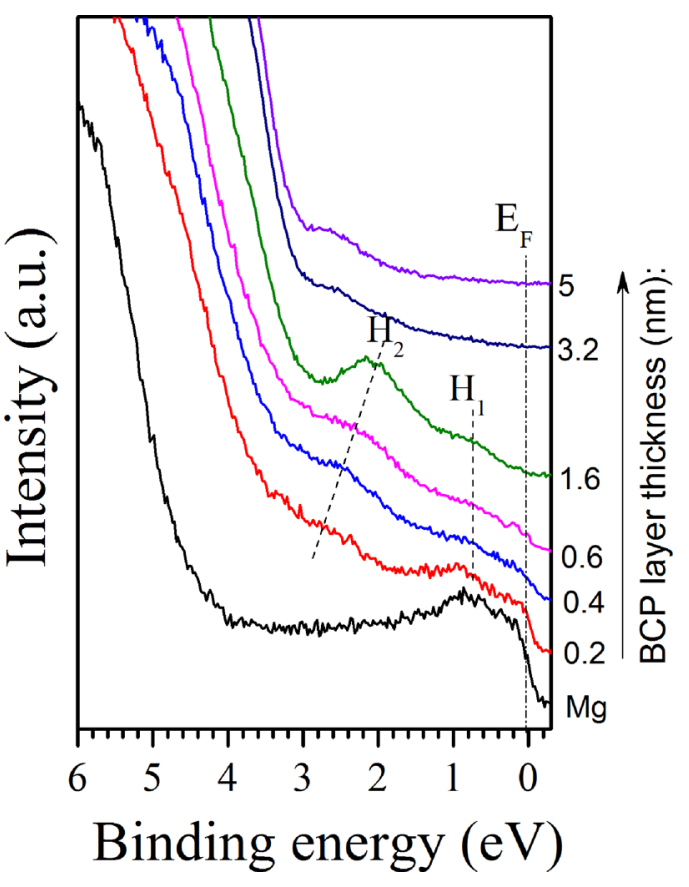

FIG. 2. UPS spectra of BCP layers with incremental thicknesses deposited on $\mathrm{Mg} / \mathrm{Si}$ substrate at the low binding energy part near the $E_{F}$.

reappearance of $\mathrm{Mg} 3 \mathrm{~s}$ peak, because clean $\mathrm{Mg} 3 \mathrm{~s}$-induced peak is located at the similar position with peak $H_{1}$. However, we cannot observe the $E_{F}$ edge from the spectra of $\mathrm{BCP}$ layer. Therefore, the reappearance of $\mathrm{Mg} 3 \mathrm{~s}$ peak in $\mathrm{BCP}$ layer can be excluded, and the peak $H_{1}$ can be assigned to new state by the interaction between BCP molecular orbital and Mg3s electrons. When organic molecule is chemically bonded to metal, the energy levels of molecule will be modified, causing shifting, broadening and the formation of new states. ${ }^{16,17}$ Therefore, the peak $H_{2}$-induced gap state may be caused by chemical bonding between BCP molecule and $\mathrm{Mg}$.

\section{B. BCP/Mg interfacial properties studied by XPS}

To derive the chemistry of $\mathrm{BCP} / \mathrm{Mg}$ interface, HR-XPS measurements were carried out, because this technique is very sensitive to the changes of the chemical environment and charge redistribution. Fig. 3(a) shows $\mathrm{C} 1 \mathrm{~s}$ core level spectra for different thickness of BCP layer. A single $\mathrm{C} 1 \mathrm{~s}$ peak was observed at $286.6 \mathrm{eV}$, which is attributed to the carbon in the benzene/pyridine backbone. ${ }^{18}$ And the $\mathrm{C} 1 \mathrm{~s}$ peak is not affected by BCP layer thickness except for some narrowing. Fig. 3(b) displays N1s core level spectra for different thickness of BCP layer. We can see that the spectra drastically changed with increasing BCP layer thickness. Two peaks at $399.8 \mathrm{eV}$ and $401.3 \mathrm{eV}$, labeled with peak $\mathrm{A}$ and peak $\mathrm{B}$, respectively, can be observed for the initial coverage (just $0.2 \mathrm{~nm}$ ), whereas there is just one peak when BCP layer thickness is up to $1.6 \mathrm{~nm}$.

From the chemical structure of BCP molecule in Fig. 1, the $\mathrm{N}$ atoms have only one chemical environment. Therefore, the N1s of isolated BCP molecule should have only one peak in XPS spectra, which was confirmed by the single peak of thick BCP layer $(3.2-5 \mathrm{~nm})$, because these 


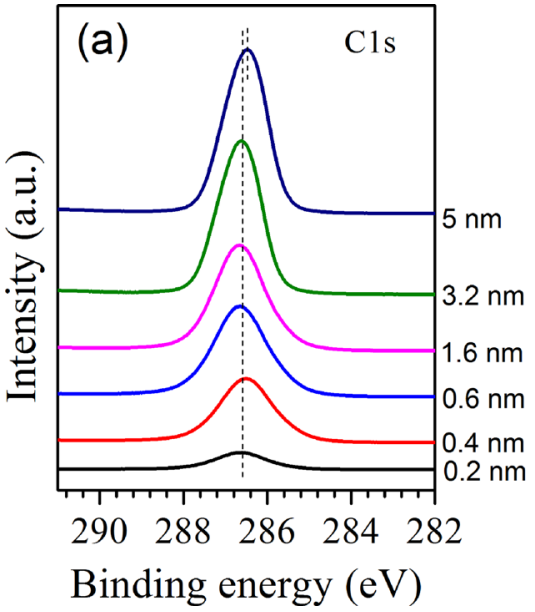

thicknesses only reflect bulk BCP structure, regardless of $\mathrm{BCP} / \mathrm{Mg}$ interface. The single peak of thick films is assigned to $\mathrm{N}$ atom emission from pyridine group. With increasing $\mathrm{BCP}$ layer thickness, the peak A shifted to higher $B E$ by $0.46 \mathrm{eV}$. This shift suggests that electron transfer takes places from $\mathrm{BCP}$ layer to $\mathrm{Mg}$, which is consistent with the dipole layer formation at $\mathrm{BCP} / \mathrm{Mg}$ interface as reported in our previous report (Ref. 14). Interestingly, the intensity of peak B gradually attenuated and this peak finally disappeared as BCP layer thickness above to $1.6 \mathrm{~nm}$. The existence of peak $B$ in the thickness range of $0.2-1.6 \mathrm{~nm}$ indicates that new component was formed at $\mathrm{BCP} / \mathrm{Mg}$ interface, which is caused by the chemical bonding between $\mathrm{N}$ atoms and $\mathrm{Mg}$ at the interface. The evolution of peak B in N1s core level spectra occurs simultaneously with the aforementioned peak $\mathrm{H}_{2}$ in valence spectra, suggesting that the peak $\mathrm{H}_{2}$-induced gap state stems from the chemical interaction at $\mathrm{BCP} / \mathrm{Mg}$ interface.

The formation of chemical bond between $\mathrm{N}$ and $\mathrm{Mg}$ atoms can be confirmed by ARPES. The ARPES measurement can probe the relative component of $\mathrm{Mg}-\mathrm{N}$ bond at interface and $\mathrm{C}-\mathrm{N}$ bond in pyridine group of $\mathrm{BCP}$ molecule.
ARPES spectra were collected at two emission angles, the surface normal and grazing emission angles $\left(90^{\circ}\right.$ and $25^{\circ}$ from the surface, respectively). The surface normal emission angle allows more bulk electrons to escape with higher intensity, while the grazing emission angle only allows surface photoelectrons to escape. So the grazing emission angle reflects much more surface information.

Fig. 4 shows ARPES spectra of N1s core level for 0.2 and $1.6 \mathrm{~nm} \mathrm{BCP}$ layer. All the spectra were normalized and fitted by Gaussian function after subtracting the Shirley background. For $0.2 \mathrm{~nm}$ BCP layer at different emission angle, the spectra shows no difference for the shape, position and relative intensity of peak $\mathrm{A}$ and peak $\mathrm{B}$, as shown in Figs. 4(a) and 4(b). This indicates that the Mg-N bond and $\mathrm{C}-\mathrm{N}$ bond components distributed uniformly because of the slight coverage of BCP. From Figs. 4(c) and 4(d), we can see that the spectra look so different for $1.6 \mathrm{~nm}$ BCP coverage. First, the ratio of relative intensity of peak $B$ and peak $A$ is drastically smaller than that of $0.2 \mathrm{~nm}$ BCP case, suggesting that the $\mathrm{Mg}-\mathrm{N}$ component reduced as compared to the $\mathrm{C}-\mathrm{N}$ bond. When BCP layer thickness is increased from 0.2 to $1.6 \mathrm{~nm}$, the $\mathrm{Mg}-\mathrm{N}$ component at the interface will be partly
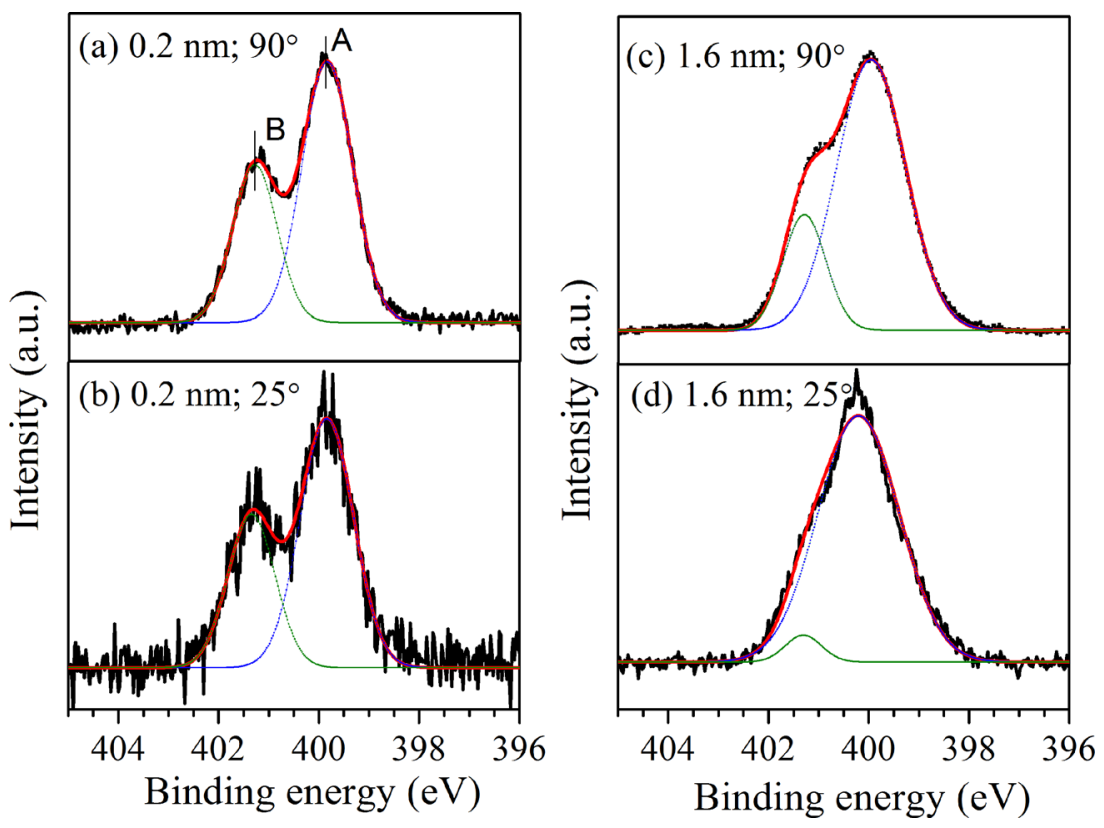

FIG. 4. X-ray photoemission spectra of $\mathrm{N} 1 \mathrm{~s}$ core levels for $(\mathrm{a}, \mathrm{b}) 0.2 \mathrm{~nm}$ and (c,d) 1.6 BCP layers detected at $(\mathrm{a}, \mathrm{c})$ surface normal emission angle $\left(90^{\circ}\right)$ and $(\mathrm{b}, \mathrm{d})$ grazing emission angle $\left(25^{\circ}\right)$. 


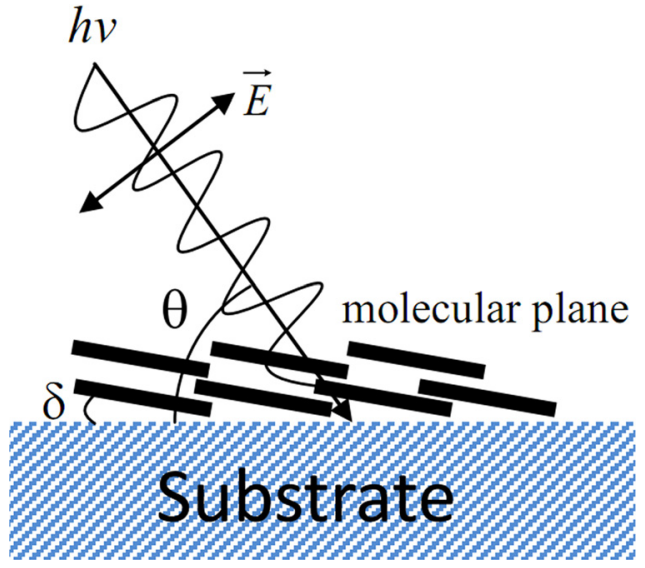

FIG. 5. Geometry of angular-dependent NEXAFS measurement. The $\delta$ and $\theta$ are the tilt angle of molecular plane and the photon incidence angle with respect to the surface plane, respectively.

suppressed and much more information from BCP layer will be detected, so the intensity of peak $\mathrm{B}$ attenuated. Additionally, the intensity of peak B for $1.6 \mathrm{~nm} \mathrm{BCP} \mathrm{layer} \mathrm{at}$ grazing emission angle is much smaller than that of surface normal emission angle. This indicates that the component of $\mathrm{Mg}-\mathrm{N}$ bond is located far away from the BCP layer surface. This again provides evidence to the formation of $\mathrm{Mg}-\mathrm{N}$ bond just at the interface.

\section{BCP molecule orientation}

To further understand the electronic structure of the $\mathrm{BCP}$ on $\mathrm{Mg}$, angular-dependent NEXAFS was carried out to characterize the molecular orientation. In principle, NEXAFS monitors the resonance from the core level of a specific atomic species of a molecule to its unoccupied molecular orbitals ( $\pi^{*}$ and $\sigma^{*}$ orbitals); the intensity of resonant peaks strongly correlated with the orientation of the $\pi^{*}$ and $\sigma^{*}$ orbitals. A strong resonant peak occurs when the electronic field vector of the incident linear polarized synchrotron light is parallel to the direction of the $\pi^{*}$ and $\sigma^{*}$ orbital, otherwise a weak peak occurs. ${ }^{19}$ Although the molecular orientation generally can be extracted by both $\mathrm{C}$ K-edge and $\mathrm{N}$ $\mathrm{K}$-edge, the $\mathrm{N}$ K-edge is much more effective for $\mathrm{BCP}$, because the $\mathrm{N}$ atoms are located at $\mathrm{BCP}$ molecular plane while the $\mathrm{C}$ atoms in pyridine group and benzene ring show different geometry. Fig. 5 shows the geometry of the angular-dependent NEXAFS measurement.

Fig. 6 displays N K-edge NEXAFS spectra for $0.2 \mathrm{~nm}$ BCP layer. The peak at $399.8 \mathrm{eV}$ and $401.5 \mathrm{eV}$ are attributed to transitions to the $\pi^{*}$ states, which are oriented perpendicularly to the molecular plane. The broad features at higher photon energy part belong to transitions to $\sigma^{*}$ states that are oriented in the plane of the molecule. We can see that the relative intensity of $\pi^{*}$ and $\sigma^{*}$ transitions varies strongly with the photon incident angle $\theta$. The intensity of $\pi^{*}$ transitions is minimum at normal incidence $\left(\theta=90^{\circ}\right)$, while $\sigma^{*}$ transition shows maximum intensity. With changing the incidence angle from normal to grazing, the intensity of $\pi^{*}$ transitions gradually increase and the intensity of $\sigma^{*}$ transitions gradually decrease. This suggests that BCP molecule shows lyingdown configuration on substrate plane.

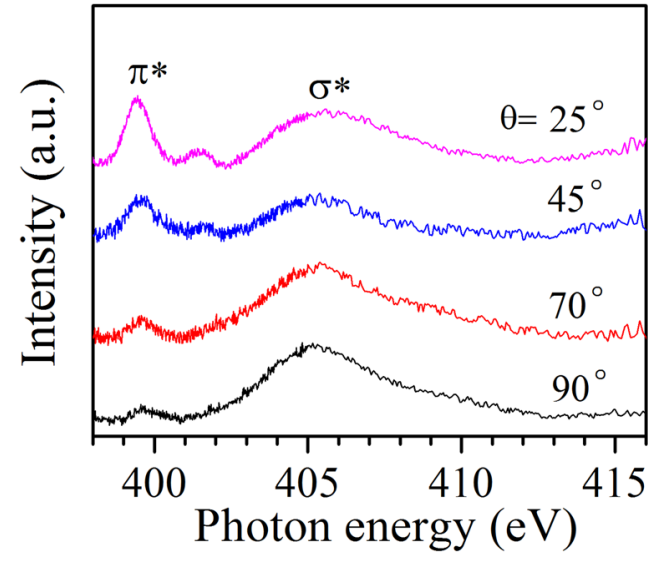

FIG. 6. N K-edge NEXAFS spectra as a function of photon incidence angle $(\theta)$ for $0.2 \mathrm{~nm}$ BCP layer. by 19

The azimuthally averaged tilt angle can be obtained

$$
\mathrm{I}(\theta) \propto 1+\frac{1}{2}\left(3 \cos ^{2} \theta-1\right)\left(3 \cos ^{2} \delta-1\right)
$$

where $I$ is the $\pi^{*}$ intensity, relating to the tilt angle $\delta$ and the photon incidence angle $\theta$. Fig. 7 is the plot of the experimental $\pi^{*}$ intensity against the calculated variation of a range of planar tilt angle. From it, the tilt angle $\delta$ was obtained to be $25 \pm 5^{\circ}$. We also got the $\mathrm{N}$ K-edge NEXAFS spectra for $0.6 \mathrm{~nm} \mathrm{BCP}$ coverage (not shown here), and the resultant spectra showed the similar tendency with $0.2 \mathrm{~nm} \mathrm{BCP}$ coverage. We suppose that the BCP molecules have the same orientation for $0.2-1.6 \mathrm{~nm}$ and it may shows different orientation above $1.6 \mathrm{~nm}$, because this thickness is the divide for the interfacial interaction as are discussed in UPS and XPS results.

To verify the deduction above, $\mathrm{N}$ K-edge NEXAFS spectra for $3.2 \mathrm{~nm}$-thick BCP was obtained, as shown in Fig. 8 , where the $\pi^{*}$ transition intensity have been normalized at the same intensity for both spectra. The peak features are different from thin BCP layer case as expected. The ratio of peak intensity of $\pi^{*}$ and $\sigma^{*}$ transitions has no any evolution with changing the incidence angle from $90^{\circ}$ to $25^{\circ}$,

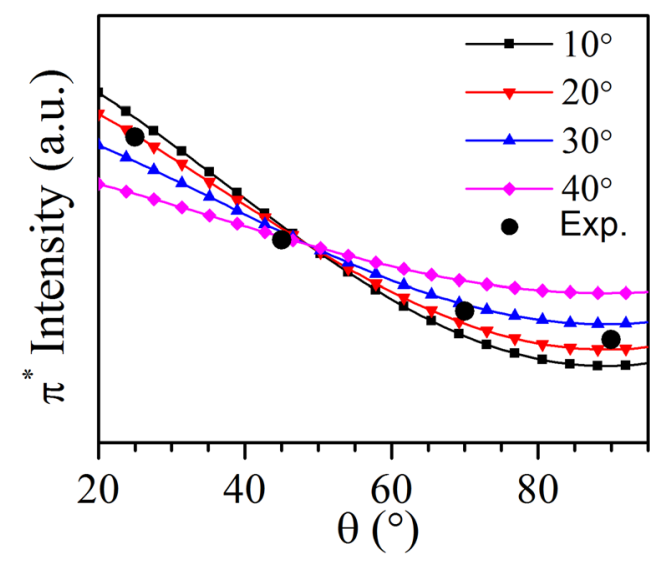

FIG. 7. Plot of the experimental $\pi^{*}$ intensity against the calculated variation of a range of planar tilt angle of BCP molecule. 


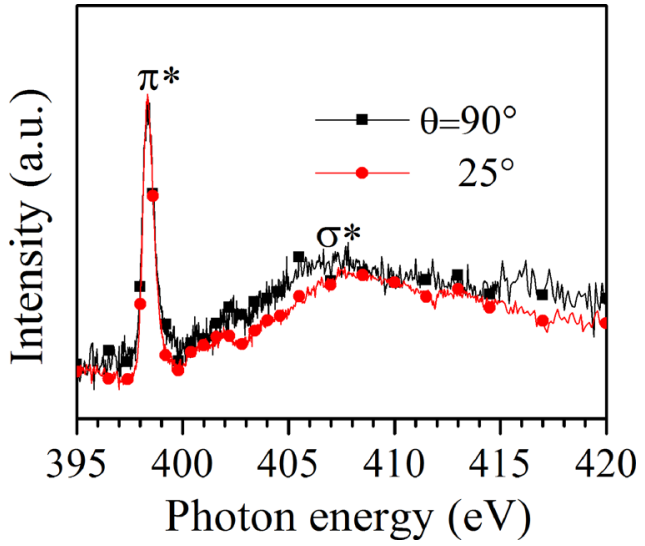

FIG. 8. N K-edge NEXAFS spectra as a function of photon incidence angle $(\theta)$ for $3.2 \mathrm{~nm}$ BCP layer.

suggesting that $\mathrm{BCP}$ molecules exhibit random orientation when the thickness above $1.6 \mathrm{~nm}$.

For BCP, the nominal thickness of $0.6-0.8 \mathrm{~nm}$ corresponds to a monolayer (ML). When BCP layer is as thin as 1-2 MLs, the electrons separated from excitons in device can be effectively extracted, because there is no barrier at $\mathrm{BCP} / \mathrm{Mg}$ interface (Ref. 14) and the gap states at this interface can provide extra channels for electrons transfer. Furthermore, the transfer speed of electron transfer will be fast because the highly-ordered molecule orientation can improve electron transport and increase mobility. ${ }^{20}$ On the contrary, for thick BCP layer case, the transfer of electrons becomes much more difficult as compared with the thin BCP layer case. The following are responsible to it. First, the large barrier at $\mathrm{C}_{60} / \mathrm{BCP}$ interface will block the electrons transfer from $\mathrm{C}_{60}$ to $\mathrm{BCP}$ layer. ${ }^{12}$ Second, the disordered orientation of BCP molecules of multiple layers also has a detrimental influence on the electrons transfer. Additionally, there is lack of transport channels for the electron because of the gap states are suppressed by thick BCP film. Thus, thin BCP layer (1-2 MLs) is beneficial to the electron transfer, and therefore can enhance the device performance. This is highly consistent with the device performance in the report of Chang et al. ${ }^{11}$ They demonstrated that the OSC with just 2 nm BCP buffer layer showed high PCE of $4.11 \%$, and the thick BCP film will lower the $P C E$.

\section{CONCLUSION}

In summary, we demonstrated that there are strong interactions between $\mathrm{BCP}$ and $\mathrm{Mg}$, resulting in a favorable electronic structure of interfaces for OSCs. Strong chemical interaction was found by forming $\mathrm{Mg}-\mathrm{N}$ bond at the interface. This interaction can produce gap states, and it can result in a highly-ordered lying-down orientation of $\mathrm{BCP}$ molecules. Both of them are beneficial to the electron transfer in OSCs. Therefore, thin BCP intermediate layer will be desirable in OSCs. When this kind of interaction is screened by thick $\mathrm{BCP}$ layer, the electron transfer from $\mathrm{C}_{60}$ layer to electrode in devices will become difficult, and therefore, being detrimental to the device performance. Thereby, the mechanism of the efficiency enhancement by thin-BCP buffer layer in OSCs was clarified. The beneficial gap states and highly-ordered orientation of BCP molecules at $\mathrm{BCP} / \mathrm{Mg}$ interface are the reasons for improving the efficiency of OSCs with BCP buffer layer and low WF metal. The present study also gives a guide to the selection of buffer layer and electrode for OSCs.

\section{ACKNOWLEDGMENTS}

This work was partially supported by the Japan Society for the Promotion of Science (JSPS).

${ }^{1}$ H. X. Wei, J. Li, Y. Cai, Z. Q. Xu, S. T. Lee, Y. Q. Li, and J. X. Tang, Org. Electron. 12, 1422 (2011).

${ }^{2}$ S. Schubert, J. Meiss, L. Müller-Meskamp, and K. Leo, Adv. Energy Mater. 3, 438 (2013).

${ }^{3}$ E. Ahlswede, J. Hanisch, and M. Powalla, Appl. Phys. Lett. 90, 163504 (2007).

${ }^{4}$ P. Peumans, V. Bulović, and S. R. Forrest, Appl. Phys. Lett. 76, 3855 (2000).

${ }^{5}$ P. Vivo, J. Jukola, M. Ojala, V. Chukharev, and H. Lemmetyinen, Sol. Energy Mater. Sol. Cells 92, 1416 (2008).

${ }^{6}$ H. Kageyama, H. Kajii, Y. Ohmori, and Y. Shirota, Appl. Phys. Express 4, 032301 (2011).

${ }^{7}$ Z. Q. Xu, J. P. Yang, F. Z. Sun, S. T. Lee, Y. Q. Li, and J. X. Tang, Org. Electron. 13, 697 (2012).

${ }^{8}$ A. R. b. M. Yusoff, H. P. Kim, and J. Jang, Org. Electron. 14, 858 (2013).

${ }^{9}$ T. Salim, Z. Yin, S. Sun, X. Huang, H. Zhang, and Y. M. Lam, ACS Appl. Mater. Interfaces 3, 1063 (2011).

${ }^{10}$ P. Peumans, V. Bulovic, and S. R. Forrest, Appl. Phys. Lett. 76, 2650 (2000).

${ }^{11}$ C. C. Chang, C. F. Lin, J. M. Chiou, T. H. Ho, Y. Tai, J. H. Lee, Y. F. Chen, J. K. Wang, L. C. Chen, and K. H. Chen, Appl. Phys. Lett. 96, 263506 (2010).

${ }^{12}$ S. Wang, T. Sakurai, R. Kuroda, and K. Akimoto, Appl. Phys. Lett. 100, 243301 (2012).

${ }^{13}$ D. Y. Luo, L. M. Yu, J. X. Man, T. L. Liu, J. J. Li, T. Xu, Z. Liu, Z. B. Wang, and Z. H. Lu, J. Appl. Phys. 113, 224506 (2013).

${ }^{14}$ T. Sakurai, S. Toyoshima, H. Kitazume, S. Masuda, H. Kato, and K. Akimoto, J. Appl. Phys. 107, 043707 (2010).

${ }^{15}$ M. D. Bhatt, S. Suzuki, T. Sakurai, and K. Akimoto, Appl. Surf. Sci. 256, 2661 (2010).

${ }^{16}$ S. Masuda, Appl. Surf. Sci. 256, 4054 (2010).

${ }^{17}$ M. Sogo, Y. Sakamoto, M. Aoki, S. Masuda, S. Yanagisawa, and Y. Morikawa, J. Phys. Chem. C 114, 3504 (2010).

${ }^{18}$ C. Grave, C. Risko, A. Shaporenko, Y. Wang, C. Nuckolls, M. A. Ratner, M. A. Rampi, and M. Zharnikov, Adv. Funct. Mater. 17, 3816 (2007).

${ }^{19}$ J. Stöhr, NEXAFS Spectroscopy (Springer, Berlin, 1992).

${ }^{20}$ K. Ahn, J. B. Kim, H. Park, H. Kim, M. H. Lee, B. J. Kim, J. H. Cho, M. S. Kang, and D. R. Lee, Appl. Phys. Lett. 102, 043306 (2013). 